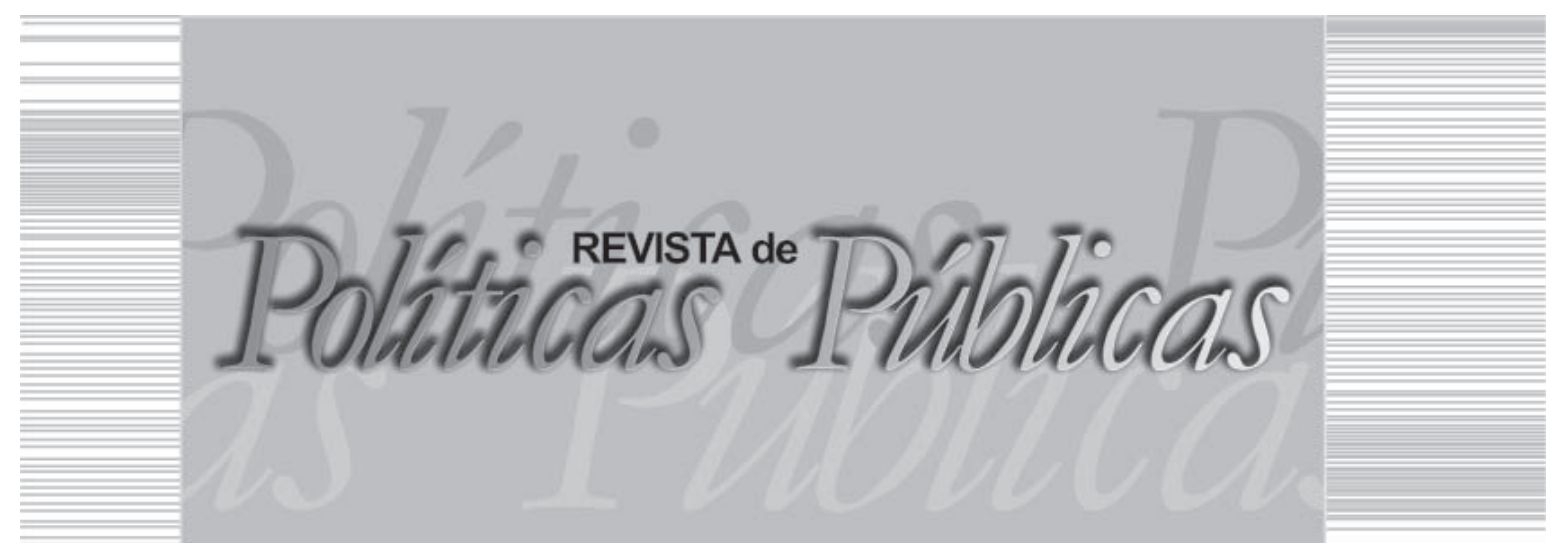

\title{
LA NUEVA GESTIÓN DE LO SOCIAL EN URUGUAY: un avance de la ideología neoliberal
}

\author{
Alejandro Gabriel Mariatti ${ }^{I}$ \\ Universidad de la República (Udelar)
}

\section{Resumen}

Desde la década de 1970, como resultado final de las tres décadas gloriosas (HOBSBWAM, 1999) y, especialmente, a partir del consenso de Washington (DOMINGUEZ UGA, 2004) la doctrina neoliberal como una ideologia de ajuste para la recomposición de la tasa de lucro (HAYEK, 2006; FRIEDMAN, 1966; GENTILI, 1997), se recomienda reducir la asistencia social a la política focalizada. La protección social y del mercado de trabajo (OFFE, 1976) se adapta a las necesidades y posibilidades de producción pos crisis, Este texto explora el proceso despolitizante (LECHNER, 2008; LUKÁCS, 2000) e individualizante (POLANYI, 1992; DANANI, 2008) que posee la política social. Lejos de reducir la acción del estado, la intervención es motivada con recriar (POLANYI, 1992) sistema de mercado de trabajo.

Palabras clave: Protección social, transferencia de renta, neoliberalismo.

THE NEW MANAGEMENT OF SOCIAL IN URUGUAI: an advance of neoliberal ideology

\begin{abstract}
Since the decade of 1970 s, as a final result of the three glorious decades (HOBSBWA, 1999) and, specially, from the consensus of Washington (DOMINGUEZ UGA, 2004) the neoliberal doctrine as an adjust ideology to the

\footnotetext{
Assistente Social. Doctorando en Ciencias Sociales. Professor del Departamento de Trabajo Social de la Universidad de la República (Udelar) | E-mail: alejandromariatti@ gmail.com | Universidad de la República - Udelar | Constituyente 1502, piso 4 (personal: Marcelino Sosa 3219), Montevideo, Uruguay.
} 
recomposition of profit taxes (HAYEK, 2006; FRIEDMAN, 1966; GENTILI, 1997). The social and job market protection (OFFE, 1976), adapts to the needs and possibilities of post crisis production. This text explores the despoliticizing (LECHNER, 2008; LUKÁCS, 2000) and individualizing process (POLANYI, 1992:257; DANANI, 2008) that possess the social politics. Far from reducing the action of the state, the intervention is motivated with the recreate (POLANYI, 1992) of the job market system.

Key words: Social Protection, income transfer, neoliberalism.

\section{INTRODUCCIÓN}

En América Latina la implementación de este ajuste se comenzó a procesar en parte por medio de la fuerza. La herencia dictatorial tuvo entre muchas consecuencias el aumento de la deuda externa que en Uruguay se multiplicó por diez (YAFFÉ, 2009) como escenario de los años ochenta. A partir de los años noventa, siguiendo esta lógica de ajuste, las políticas sociales presentaron características neoliberales (GRASSI, 2006; MIDAGLIA, 1998), como consecuencia de las referidas recomendaciones. En Uruguay, en dicha década, se creó la Oficina de Planeamiento y Presupuesto, que sirvió de by-pass para dichas políticas (MIDAGLIA, 1998). Ya entrado el siglo XXI y con un cambio de rumbo en los gobiernos regionales a partir de la llegada de distintas fuerzas progresistas, esta receta sorprendentemente parece repetirse. Se implementaron una serie de experimentos (BENTURA; VECINDAY, 2010) con tautológica evaluación (BENTURA; VECINDAY, 2013), anclaje territorial (BARAIBAR, 2005) que actuaron como mecanismos privatizadores, individualizantes y comunitarizantes (DANANI, 2008) y con fuerte sesgo punitivo (WAQUANT, 2010).

\section{LA CREACIÓN DE INSTITUCIONES ESPECÍFICAS}

Para su implementación muchos países instauraron ministerios, instituciones u oficinas con pertinencia específica. Esta creación le dio en algún sentido continuidad al proceso iniciado en los años noventa. El enfoque que portan intenta legitimar una explicación despolitizada del pauperismo, descomponiéndolo en problemas individuales, parciales, con diferentes características o dimensiones.

$[\ldots]<$ la cuestión social $>$ se fragmenta en una serie de $<$ problemas sociales $>$, con la intención de hacerla desaparecer. A cada uno de estos problemas debe corresponder un ámbito de saber, una 


\section{LA NUEVA GESTIÓN DE LO SOCIAL EN URUGUAY. UN AVANCE DE LA IDEOLOGÍA NEOLIBERAL}

especialidad profesional y unas técnicas específicas de intervención. De este modo, se autonomizan por ejemplo los problemas del alcoholismo, la tuberculosis, la escolarización, el aprendizaje, la vivienda, el urbanismo y el desempleo. (TOPALOV, 1990, p. 53, grifo del autor).

\section{LOS PROGRAMAS DE TRANSFERENCIA DE RENTA CONDICIONADA: en adelante PTRC}

Con relación a estos programas, América Latina tiene varios antecedentes ${ }^{1-2}$. En Uruguay orientan y definen la admisión por medio de un índice conocido como de Carencia Críticas (en adelante ICC) (URUGUAY, 2013a), que fue creado en 2008 a solicitud del Ministerio de Desarrollo Social (en adelante Mides) y al que de alguna manera ya se hacía referencia en la Ley de creación ministerial ${ }^{3}$. El Índice reviste una supuesta objetividad técnica, donde expertos (OFFE, 1976; LECHNER, 2008) establecen el camino para identificar a los beneficiarios. Hay un imbricado proceso entre dos características sobresalientes que forman parte de los Programas de Transferencia de Renta Condicionada (PTRC) del Mides, en primer lugar la optimización de los recursos (eficacia y eficiencia (OFFE, 1976) a partir de la incorporación de expertos ${ }^{4}$ capaces de identificar el límite de la asistencia; en segundo lugar la tecnificación e informatización de los dispositivos de gestión social junto a la incorporación de nuevas profesiones para materializar y concretar esta justicia experta. Este proceso reifica la realidad, otorgando un lugar hipostasiado a la admisión, ajeno a la humanidad. A su vez, la decisión experta y el algoritmo legitiman la identificación del problema como individual, exiliando cualquier interpelación. En palabras de un ex director de Mides que le explica a una señora como se desarrolla este mágico proceso "[...] se entran los datos suyos a una computadora y ésta dice si su hogar va o no va, de acuerdo al índice Crítico, después del estudio de todo lo que se le preguntó a usted con la visita, $[\ldots]$ Yo le explico, es una máquina, ¿entiende?" (MARSIGLIA, 2006), Ideológicamente, la impronta tecnocrática es una mediación para legitimar el criterio de discriminación y naturalizar la desigualdad. 


\section{LOS PROCESOS DE INDIVIDUALIZACIÓN Y DESPOLITIZACIÓN}

La tarea de visitar todos los hogares para encontrar a los beneficiarios obligó en la ejecución de los PTRC a sofisticar la metodología de admisión y control.

Esto implicó la tecnificación de los procedimientos para la
georreferenciación de los hogares, la informatización del
relevamiento de datos y la interoperabilidad entre distintos
sistemas de información. La informatización de los procesos
significó el desarrollo de un sistema de información (Q-flow) ágil,
dinámico y altamente tecnificado, que permitiera el seguimiento
del trabajo y el rápido procesamiento de la información para el
objetivo de base y para la generación de insumos para la política
social. (URUGUAY, 2013b, p. 108).

El diagnóstico se realiza hogar por hogar, suponiendo que es posible una comprensión de tal situación como aislada del resto, valorándola frente al ICC. "Mientras las leyes científicas ignoran al individuo concreto, las normas que las ciencias permiten establecer reconstruyen a un individuo diferente, que se convierte en sujeto de la administración". (TOPALOV, 1990, p. 61). El MIDEs, al momento de asignar o no un beneficio, construye una explicación oficial de beneficiario, que porta un problema personal. Naturaliza la desigualdad propia del orden burgués. "Naturalmente, hay que decidir quiénes son los que merecen la ayuda y quiénes son los que no la merecen, sin hacer intervenir la incertidumbre propia de una decisión individual." (TOPALOV, 1990, p. 63-64). El acto de identificar a un beneficiario al mismo momento explica el problema como un accidente biográfico, quitándole sociedad. De ese modo cae el telón de fondo de la lucha de clases y se promueve una explicación individual que responsabiliza al sujeto. Una realidad (desigual) naturalizada que en los términos de Lechner (2008), suponen momentos discontinuos que adquieren continuidad por medio del Estado.

De esta forma, el orden burgués es reificado, presentado como a-histórico, colocando al mercado como representante de una relación pre-social (LECHNER, 2008) y reduciendo las relaciones sociales a relaciones puramente mercantiles. El mercado es reificado como espacio natural de socialización, que explica su devenir de forma individual, solamente como propietarios de sí mismo (CASTEL, 2003). El Estado logra darle continuidad 


\title{
LA NUEVA GESTIÓN DE LO SOCIAL EN URUGUAY. UN AVANCE DE LA IDEOLOGÍA NEOLIBERAL
}

como expresión normativo-simbólica (LECHNER, 2008). En esta dimensión normativo-simbólica, los sujetos se construyen en el reciproco establecimiento de límites. "En ese doble proceso de descomposición y recomposición de las representaciones y, según se espera, de la realidad aparece una novedad de peso, y es que la ciencia y la administración, estrechamente asociadas, empiezan a desempeñar un papel esencial". (TOPALOV, 1990, p. 54). Al desplegarse la visita, surge la representación como interrogante, de ser o no ser un beneficiario. Las preguntas parecen portar la intención de husmear de forma vejatoria la intimidad del hogar, pretendiendo entender y diagnosticar desde características o indicadores parciales del entorno familiar inmediato. El techo de la casa, el saneamiento, la presencia de niños, de empleado público o de calentador de agua, son algunos valores de referencia en las visitas realizadas. La mirada individualizante supone que las claves para justificar el beneficio remiten antes que nada a una pobreza reconstruida como fracaso personal en el mercado y no como heterodeterminación propia del metabolismo del capital.

\begin{abstract}
[...] la gestión <individualizada> de los problemas sociales encuentra su condición de posibilidad en los más recientes avances tecnológicos acaecidos en el campo de la informática y en la introducción del gobierno electrónico y de las denominadas TIC. Los avances en la informática ofrecen la posibilidad técnica de construir sistemas de información cada vez más sofisticados que serán demandados por un Estado que ha burocratizado la gestión de lo social. (DE MARTINO; VECINDAY, 2011, p. 39, grifo del autores).
\end{abstract}

\section{NEO POSITIVISMO}

La instrumentalidad medios-fines despliega un doble mecanismo de control para encontrar al beneficiario, que, habiendo sido previamente georeferenciado, recibe una visita que evalúa si presentan en ese momento aquellos atributos para la admisión. Desde este mecanismo se puede saber en tiempo real quién está cobrando el beneficio, a quién le corresponde tenerlo y a quién correspondería quitárselo si no tuviere al día las condicionantes. Esta omnipresencia, le da por momentos, un bies similar a las fantasías de Orwel $^{5}$ o Huxley 6 .

En relación a la política, Lechner (2008) ubica la utilidad del cálculo racional e instrumental en la decisión política, como 
cálculo de correlación de fuerzas. Estas reflexiones, aproximan a un cálculo de la acción del otro, a su previsibilidad. Anticipar la acción del otro, calcular su devenir con rigor técnico-instrumental, e inclusive apropiarse de métodos instrumentales para gobernar al otro, "[...] hago del otro un objeto [...] La política es concebida a la imagen del trabajo del hombre sobre la naturaleza; esto es, como una acción fundamentalmente instrumental." (LECHNER, 2008, p. 6-7). Los sujetos se construyen en el vínculo con los límites, el conflicto y la negociación, donde uno se afirma en relación a un otro. "La constitución de los sujetos es un proceso fundamentalmente ideológico [...]" (LECHNER, 2008, p. 8). Esa ideología se legitima por medio de los mitos sobre la propiedad privada expresada en la doctrina del derecho iusnaturalista y la interpretación neo-clásica de una economía como separada de la organización social, suponiendo relaciones pre-sociales, traídas desde la ideología, en el sentido que le otorga Lechner $(2008$, p. 11, grifo del autor) cuando indica que "[...] el proyecto neoconservador pretende $<$ descolgar $>$ el sistema económico de la dimensión normativo-simbólica."

Los mecanismos de focalización en la actualidad, al querer encontrar al beneficiario con perfil tan especifico también parecen necesitar hacer explícita la distinción, amedrentando al candidato desde la vergüenza pública. "Se empieza a distinguir entre <clase obrera respetable $>$ y masas empobrecidas, a las que se clasifica progresivamente en categorías, cada una de ellas sujeta a un tratamiento particular y adaptado a su situación." (TOPALOV, 1990, p. 53, grifo del autor). La selección exhaustiva, la divulgación mediática de las acciones ministeriales por medio de la web institucional, el gueto construido en torno al ICC, fueron definiendo un lugar de evidencia y estigma.

La pertinencia de un Ministerio de Desarrollo Social como institución especializada, supone el surgimiento de un nuevo problema ajeno al capitalismo, que para la gestión social es construido como un problema personal, dejando el Ministerio de Trabajo para los asuntos que aún se resuelven entre el capital y el trabajo. Por eso es necesario un algoritmo matemático, presentado y aplaudido como instrumento neutro, experto, secular, transparente y despolitizado, que separa tecnocrática y centralizadamente, los admitidos de los desestimados. 


\title{
LA NUEVA GESTIÓN DE LO SOCIAL EN URUGUAY. UN AVANCE DE LA IDEOLOGÍA NEOLIBERAL
}

\begin{abstract}
Los <problemas sociales> así construidos por los nuevos profesionales adquieren la calidad de realidades objetivas, como puede comprobar cualquier mente libre de prejuicios; de este modo quedan despolitizados y escapan al ámbito de las controversias ficticias y peligrosas del enfrentamiento democrático. En poco tiempo, personajes situados en puntos diferentes e incluso opuestos del abanico político o social adoptarán un lenguaje común que delimitará el terreno de sus enfrentamientos. (TOPALOV, 1990, p. 57, grifo del autor).
\end{abstract}

El neopositivismo será la herramienta estadística para la administración y manipulación del caudal de información que la nueva incorporación tecnológica genera. "La estadística oficial acumula datos y afina sus métodos en las nuevas administraciones laborales y de salud pública, mientras el urbanismo se afirma como disciplina y profesión en el marco de las municipalidades o de los grupos cívicos locales." (TOPALOV, 1990, p. 54). Esta aparente neutralidad no es otra cosa que una mirada arbitraria sobre la realidad. La ampliación y unificación, como ciencia única, en el terreno matemático refina la apariencia de objetividad e imparcialidad. El "[...] entero sistema de saber es elevado a la condición de instrumento de una manipulabilidad general de todos los hechos relevantes." (LUKÁCS, 2012, p. 58).

\section{ASISTENCIA PUNITIVA}

Los PTRC de Mides incorporan un sistema de admisión ajeno a los operadores, técnicos, jefes y directores de programas, hipostasiando la gestión. Los saberes expertos, la articulación entre nuevas profesiones, la tecnología informacional y el algoritmo entre otros elementos, funcionaron como mecanismos que distanciaron la discrecionalidad (esto puede haber ocurrido por una motivación electoral) y a la vez reforzaron la legitimidad por medio de esta supuesta objetividad técnica, como mito de una justicia asistencial. Adjudica y ubica al mismo tiempo el lugar inamovible dentro del orden, entendiendo la pobreza como fenómeno individual, pero sobre todo interviniendo en la población para salvaguardar la creación de un mercado de mano de obra. El triunfo hegemónico del neoliberalismo, profundiza la reificación del orden burgués y complementando la asistencia con control punitivo ${ }^{7}$ (WACQUANT, 2010). En algún sentido, las reflexiones de Topalov (1990, p. 60) guardan similitud con las de Wacquant, a pesar de referirse a procesos 
temporales distintos, "[...] es posible que afloren los métodos represivos hasta hacerse visibles de manera permanente en algunos sectores de la población y en algunas coyunturas de importantes crisis a más amplia escala."

Como el pauperismo va de la mano de la abundancia, para el orden burgués, es necesario administrarlo en tanto éste representa parte del proceso de construcción del mercado de mano de obra y la conversión de trabajo en mercancía (POLANYI, 1992). En los albores de la revolución industrial, el trabajo como actividad humana unida a la vida misma fue dislocado catastróficamente y convertido en mercancía de forma ficticia. "El liberalismo económico leyó mal la historia de la Revolución industrial porque insistía en juzgar los eventos sociales desde el punto de vista económico." (POLANYI, 1992, p. 45). Ese pauperismo que acompaña al capitalismo desde sus inicios ha sido siempre objeto de administración, "[...] resultaba muy importante que tal fondo de mano de obra móvil estuviese a disposición de la industria en período de expansión." (POLANYI, 1992, p. 142), entre otras cosas, porque "[...] el mercado ha derivado en una intervención consciente y a menudo violenta del gobierno, que impuso la organización del mercado a la sociedad por razones no económicas.” (POLANYI, 1992, p. 248).

\section{LA ETERNIZACIÓN DEL MERCADO Y LOS NUEVOS EMPLEOS}

La preocupación de orden de la ideología neoliberal es colocar al mercado como la única opción de vida. Esto, como decíamos al inicio, implicó procesos de intervención, puesto que "[...] los mercados libres no podían haber surgido jamás con solo permitir que las cosas tomaran su curso." (POLANYI, 1992, p. 144). Muy similar a lo que ocurría a fines de siglo XIX en Uruguay y como consecuencia del alambramiento del campo, "[...] la permanente persecución de los vagos, iya no se puede vivir sin trabajar!" (JACOB, 1969, p. 100), determinando la creación de un mercado de mano de obra. "El camino hacia el mercado libre se había abierto y se mantenía abierto por un incremento enorme del intervencionismo continuo, centralmente organizado y controlado." (POLANYI, 1992, p. 146). De alguna forma lo que nos dice el autor es que el laissezfaire nada tiene que ver con el liberalismo en tanto este último apela a la intervención para favorecer la creación de un mercado 


\section{LA NUEVA GESTIÓN DE LO SOCIAL EN URUGUAY. UN AVANCE DE LA IDEOLOGÍA NEOLIBERAL}

de mano de obra. La novedad en este siglo XXI es representada por la incorporación de tecnología informática y sistemas expertos de búsqueda y manejo de datos para administrar la llamada cuestión social (PAULO NETTO, 2003). Dicho control está sujeto al cuidado de los equilibrios macro económicos, ubicando en un rol central a la economía y la informática. Se erigen dispositivos científicos (BORON, 2005) pseudo objetivos (LUKÁCS, 2000) con la intención de neutralizar la acción política de las clases subalternas y como mecanismo irracional (LUKÁCS, 2000) que oculta las secuelas del capitalismo. El marco ideológico de referencia será la preocupación por la equidad y la igualdad de oportunidades (DANANI, 2008). La creación de estos programas contó en Uruguay con mayoría parlamentaria de un gobierno progresista, por tanto fue un límite auto-impuesto (BENTURA et al., 2011).

Si comparamos hacia atrás, así como el inicio del siglo XX nos muestra un estado preocupado por construir al salariado, la lógica de inicio de siglo XXI parece ser la de construir al salariado-intermitente. Este mercado de mano de obra precarizado es sostenido en parte por inversiones que generan empleos de baja remuneración e intermitencia contractual (empresas de seguridad, servicios turísticos, servicio doméstico y supermercados) ${ }^{8}$ y por otra parte, la socialización del costo de su reproducción por medio de los PTRC. Las palabras de un ex Ministro son elocuentes en este sentido, "Que más de un 70\% de los tarjeta habientes trabajan lo he dicho reiteradas veces [...]" (OLESKER, 2016). Vale aclarar que el entrevistado se refiere a aquellos que además de tener la admisión del ICC para obtener el beneficio de Asignación familiar, también, tienen la aprobación aún más focalizada para obtener un beneficio económico extra en una tarjeta magnética (TUS) que se recarga mensualmente.

De esa forma, queremos expresar como es creado por medio de la intervención estatal un mercado de mano de obra intermitente. En lugar de soñar "[...] con un obrero nuevo, estabilizado en el empleo asalariado, móvil en un espacio urbano ampliado y cuyos modos de consumo tengan como único fin la productividad." como dice Topalov (1990, p. 66), la economías actuales que poseen una oferta laboral heterogeneizada, precarizada e intermitente, como consecuencia de la metamorfosis del mundo del trabajo post crisis, sueñan con un sujeto individualizado, capaz de entrar y salir al mercado de trabajo, a partir de nuevas versiones de trabajo parcial, 
conforme a las necesidades de ajuste de la acumulación flexible (HARVEY, 1992). Por esta razón, se diseñan políticas sociales capaces de encontrar en el momento justo al beneficiario.

\section{CONCLUSIONES}

Los PTRC del Mides promueven una interpretación de un pauperismo individual. Una construcción del sujeto beneficiario como portador de una miseria propia, sufrida como consecuencia de su irresponsabilidad. La individuación es el ropaje despolitizante de la ideología neoliberal en las nuevas políticas sociales. No solo entiende la pobreza como fenómeno individual sino que promueve en los beneficiarios una interpretación individual del problema, lo que la hace doblemente despolitizante. La individuación promovida por los PTRC está acompañada de un fuerte proceso de control sobre las conductas personales. El control sobre las contrapartidas permite el monitoreo personal, reuniendo en todo momento las potestades para la suspensión del beneficio (MIDES..., 2014) ${ }^{9}$. Este control infantiliza a las poblaciones asistidas al tiempo que expresa un perfil punitivo en su desempeño, colocando como tema policiaco la llamada cuestión social. La creación de acciones conjuntas del Mides con el Ministerio de Interior en Uruguay, avizoran una nueva modalidad que emparenta pauperismo con delincuencia y atiende las situaciones con asistencia y mano dura. El neoliberalismo se consolidó como ideología capaz de explicar la pobreza en sí misma, por fuera de la lucha de clases, incluso desde gobiernos llamados progresistas. Tomando distancia del enfoque que reivindicaba la igualdad, devienen en una preocupación por la equidad en las oportunidades para desiguales competidores (DANANI, 2008), con una desigualdad naturalizada. Su lógica es la mercantilización de todas las relaciones sociales y por medio de la intervención crear un mercado de mano de obra intermitente. Este proceso está dando forma a un nuevo modelo de gestión de lo social en Uruguay.

\section{REFERENCIAS}

ASOCIACIÓN DE TRABAJADORES DE LA SEGURIDAD SOCIAL. Salarios sumergidos en 2014: diagnóstico de cara a los próximos Consejos de Salarios. Montevideo, 2014. Disponible en: $<$ http://www.atss.org.uy/analisis-economico/salarios-sumergidos- 


\section{LA NUEVA GESTIÓN DE LO SOCIAL EN URUGUAY. UN AVANCE DE LA IDEOLOGÍA NEOLIBERAL}

en-2014-diagnostico-de-cara-a-los-proximos-consejos-de-salarios/>. Acceso en:

BARAIBAR, X. Tan cerca, tan lejos: Acerca de la relevancia "por defecto" de la dimensión territorial. Revista Fronteras, Montevideo, n. 5, p. 59-72, 2005.

BENTURA, J. P.; MARIATTI, A. Orientación y límites a la intervención del estado: la integración como demarcación de la intervención asistencial. In: JORNADAS DE INVESTIGACIÓN DE LA FACULTAD DE CIENCIAS SOCIALES, 10., 2011, Montevideo. Anais... Montevideo: UdelaR, 2011.

; VECINDAY, L. El desarrollo de experimentos de inserción como respuesta ante los problemas de integración social. Revista Trabajo Social Antioquia, Medellín, n. 10, p. 127-146, 2010.

; VECINDAY, L. La evaluación <tautológica> de los programas de transferencia de renta condicionada. Revista Políticas públicas, São Luis, v. 17, n. 1, 2013.

BORON, A. Las ciencias sociales em la era neoliberal: entre la academia y el pensamiento crítico. In: CONGRESO DE LA ASOCIACIÓN LATINOAMERICANA DE SOCIOLOGÍA, 25., 2005, Porto Alegre. Anais... Porto Alegre, 2005. Conferencia magistral.

CASTEL, R. Propiedad privada, propiedad social, propiedad de si mismo: conversasciones sobre la contrucción del individuo moderno. Rosario: Homo Sapiens, 2003.

DANANI, C. América Latina luego del mito del progreso neoliberal: las políticas sociales y el problema de la desigualdad. Ciências Sociais Unisinos, São Leopoldo, v. 44, n. 1, p. 39-48, jan./abr. 2008.

DE MARTINO, M.; VECINDAY, M. L. Notas sobre nuevas formas de gestión de la pobreza: individuación, informatización y responsabilidad familiar de los problemas sociales. Revista Tendencia y Retos, Colombia, n. 16, p. 33-42, nov. 2011.

DOMINGUEZ UGA, V. A categoria "pobreza" nas formulações de política social do Banco Mundial. Revista Sociologia Política, Curitiba, n. 23, p. 55-62, 2004.

FRIEDMAN, M. Capitalismo y libertad. Madrid: Rialp, 1966.

GALLARDO, J; GARCE, A; RAVECCA, P. Think tanks y expertos en el gobierno del Frente Amplio (Uruguay, 2005-2008). Documento online, n. 1/9, jul. 2009. 
GENTILI, P. La trama del neoliberalismo: mercado, crisis y exclusión social. Buenos Aires: Universidad de Buenos Aires: Oficina de Publicaciones del C.B.C., 1997.

GRASSI, E. Políticas y problemas sociales en la sociedad neoliberal. Buenos Aires: Espacio Editorial, 2006.

HARVEY, D. Condição Pós-Moderna. São Paulo: Edições Loyola, 1992.

HOBSBAWM, E. Historia del siglo XX. Buenos Aires: Critica, 1999.

JACOB, R. Consecuencias sociales del alambramiento (1872-1880). Montevideo: Ed. Banda Oriental, 1969.

LECHNER, N. Especificando la política. [S. 1.], 2008. Mimeo.

LUKÁCS, G. La crisis de la filosofía burguesa. [S. 1.]: Elaleph, 2000. 2012. . Para uma ontología do ser social I. Sao Paulo: Boitempo,

MARSIGLIA, D. [Encargado de la Unidad de Seguimiento del MIDES]. Diario El Serrano, Minas, 2006.

MIDAGLIA, C. El rendimiento de los 'By-pass' como instrumento de reforma social: el caso PRIS. Revista Uruguaya de Ciencia Política, Montevideo, v. 10, p. 79-100, 1998.

MIDES admite que da tarjetas sociales a personas que nos las precisan. El Pais, Montevideo, 2014. Uruguay Social. Disponible en: $<$ http://www.elpais.com.uy/informacion/mides-admite-que-datarjetas.html $>$. Acceso en:

OFFE, C. La política social y la teoría del Estado. [S. 1.: s. n.], 1976. Mimeo.

OLESKER, D. Entrevista: En ocho años, los beneficiarios de la tarjeta del Mides se redujeron a la mitad. Montevideo: PS90, 2016. Disponible en: $<$ http://ps.org.uy/?Q=articulo\&ID=3822>. Acceso en:

PAULO NETTO, J. Cinco notas a propósito de la "cuestión social". In: BORGIANNI, E.; GUERRA, Y.; MONTAÑO, C. (Orgs.). Servicio social crítico. São Paulo: Cortez, 2003.

POLANYI, K. La gran transformación crítica del liberalismo económico. Madrid: La piqueta, 1992.

SILVA, M. O. da S. e. et al. Protecao social e programas de transferencia de renda na America Latina e Caribe. In: JORNADAS 


\section{LA NUEVA GESTIÓN DE LO SOCIAL EN URUGUAY. UN AVANCE DE LA IDEOLOGÍA NEOLIBERAL}

INTERNACIONAL DE POLÍTICAS PÚBLICAS, 6., 2013, São Luís. Anais... São Luís: PPGPP/UFMA, 2013.

TOPALOV, C. De la "cuestión social" a los "problemas urbanos": los reformadores y la población de las metrópolis a principios de siglo XX. Revista Internacional de Ciencias Sociales, [S. 1.], n. 125, p. 4171, sept. 1990. (Reproducido con la autorización de la UNESCO).

URUGUAY. Ministerio de Desarrollo Social. Dirección Nacional de Evaluación y Monitoreo. ¿Que es el índice de carencias críticas? Montevideo, 2013a. (Aportes a la conceptualización de la pobreza y la focalización de las políticas sociales en Uruguay). Disponible en: $<$ http://dinem.mides.gub.uy/innovaportal/file/35169/1/que_es_el_ indice_de_carencias_criticas_-_daes_-_desytc_-_dinem.pdf $>$. Acceso en: 1 dez. 2016.

URUGUAY. Ministerio de Desarrollo Social. Dirección Nacional de Evaluación y Monitoreo. Informes MIDES: seguimiento y evalución de actividades y programas 2011-2012. Montevideo, 2013b. Disponible en: $<$ http://dinem.mides.gub.uy/innovaportal/file/35169/1/ que_es_el_indice_de_carencias_criticas_-_daes_-_desytc_-_dinem. pdf`. Acceso en: $\overline{1}$ dez. 2016.

WACQUANT, L. Castigar a lós pobres. Barcelona: Editorial Gedisa, 2010 .

\section{NOTAS:}

1 Repasamos algunos de ellos: Programa de Asignación Familiar (PRAF), Bonos 10.000 Programa Presidencial Salud, Educación y Nutrición en Honduras, Oportunidades en México, Programa Familias en Acción en Colombia, Programme of Advancement through Health and Education PATH en Jamaica, Chile Solidario en Chile, Bolsa Familia en Brasil, Red Solidarieda El Salvador, Red Juntos en Perú, Programa Abrazo, Programa Tekoporã y Programa Ñpytyvô en Paraguay, Programa Solidaried en R. Dominicana, Red de Oportunidades en Panamá, Avancemos en Costa Rica, Target Conditional Cash Transfer (TCCTP) en Trinidad y Tobago, Programa Nuevo Régimen de Asignaciones Familiares - AFAM-PE en Uruguay, Programa Bono Madre - Niño Juana Azurduy en Bolivia, Programa Asignación Universal por Hijo en Argentina y Mi Bono seguro en Guatemala. (SILVA et al., 2013).

2 Se puede ampliar la información con las investigaciones realizadas por el equipo de Estudio comparado de programas de transferencia de renta condicionada en Uruguay - Ingreso ciudadano - Asignaciones Familiares con otros programas de similares características del continente. Se trata de un proyecto de investigación conjunto enmarcado en el Programa CAPES PPCP - MERCOSUL (Edital CGCI n. 072/2010, Proceso Selectivo 2010/2011) y MERCOSUR EDUCATIVO de la ANIII.

3 Inciso e articulo 9 de la Ley n. 17866, de 31 de marzo de 2005. 
4 “[...] las principales políticas implementadas han tenido un fuerte componente técnico. Tanto el Plan de Emergencia como el Plan de Equidad fueron diseñados e implementados con un fuerte respaldo de expertos en políticas sociales." (GALLARDO; GARCE; RAVECCA, 2009, p. 24-25)

5 Libro: 1984

6 Libro: Un mundo feliz

7 VerlanzamientodePlan(Disponibleen:<http://www.mides.gub.uy/innovaportal/v/23894/3/ innova.front/lanzamiento-del-plan-siete-zonas >).

8 "En 2014, unos 690.000 ocupados percibieron remuneraciones líquidas por debajo de los $\$ 15.000$ mensuales [500 dólares americanos para 2015], representando $41,5 \%$ del total de ocupados ... Los sectores más afectados son el servicio doméstico con casi $63 \%$ de trabajadoras con salarios que no alcanzan los 15.000 líquidos". (ASOCIACIÓN DE TRABAJADORES DE LA SEGURIDAD SOCIAL, 2014).

9 (Palabras del Sub Secretaria de Mides): El año pasado el Ministerio de Desarrollo Social hizo "[...] 67.000 visitas para la refocalización de la tarjeta 'Uruguay Social', las visitas las hacemos todos los años porque entendemos que cada dos años la información puede modificarse, el contexto en el cual la familia se maneja. Y en esas revisiones hay caídas y subidas de categorías porque tenemos la tarjeta simple y la doble.", afirmó Meléndez. De estas visitas resultaron aproximadamente 9.000 altas y 13.500 bajas, según informa el Mides (MIDES..., 2014). 